\title{
Actualización en el manejo agudo de errores congénitos del metabolismo
}

\author{
PAULINA BRAVO J. ${ }^{1}$, GABRIELA CASTRO $\mathrm{CH}^{2}{ }^{2}$ \\ 1. Pediatra, Nutrióloga. Laboratorio de Genética y Enfermedades Metabólicas (LabGEM). \\ Instituto de Nutrición y Tecnología de los Alimentos (INTA). Universidad de Chile. \\ 2. Nutricionista. Laboratorio de Genética y Enfermedades Metabólicas (LabGEM). \\ Instituto de Nutrición y Tecnología de los Alimentos (INTA). Universidad de Chile.
}

\begin{abstract}
Update on acute management of inborn errors of metabolism

Inborn metabolic disorders are genetic diseases which are uncommon each one, but together they are not. They are characterized by an enzimatic defect that blocks a metabolic pathway, producing specific signs and symptoms. The current article pretends be an updated guideline for their acute management which is based on: 1) Inmediate life support, hydroelectrolyte balance and sample procurement, 2) Avoiding the production of toxic endogenous metabolites and anabolism promotion, 3) The supplementation of substrates and 4) The removal of toxic substances. Their prompt suspicious, identification and treatment starting will be crucial for neurological prognosis and prevention of death.
\end{abstract}

(Key words: Inborn error of metabolism, enzyme disorder, acute management, hyperammonemia).

Rev Chil Pediatr 2014; 85 (4): 421-427

\section{RESUMEN}

Los errores congénitos del metabolismo son enfermedades genéticas poco frecuentes pero que en conjunto no lo son. Se caracterizan por un defecto enzimático que bloquea una vía metabólica produciendo manifestaciones clínicas características. El objetivo del presente artículo es presentar una guía actualizada para su manejo agudo, el cual consiste en: 1) Medidas inmediatas de soporte vital, manejo hidroelectrolítico y obtención de muestras críticas; 2) Evitar la producción endógena de metabolitos tóxicos y favorecer el anabolismo; 3) Suplementación de sustratos y 4) Remoción de sustancias tóxicas. Su pronta sospecha, identificación e inicio de tratamiento, resulta fundamental para mejorar el pronóstico neurológico y evitar la muerte.

(Palabras clave: Error innato del metabolismo, desorden enzimático, manejo agudo, hiperamonemia).

Rev Chil Pediatr 2014; 85 (4): 421-427

Recibido el 07 de julio de 2014, aceptado para publicación el 01 de agosto de 2014.

Potenciales conflictos de interés: Este trabajo cumple con los requisitos sobre consentimiento /asentimiento informado, comité de ética, financiamiento, estudios animales y sobre la ausencia de conflictos de intereses según corresponda. 


\section{Introducción}

Los errores congénitos del metabolismo (ECM) son un grupo heterogéneo de enfermedades poco frecuentes. La mayoría se reconocen en la edad pediátrica, desde las primeras horas de vida hasta la adolescencia ${ }^{1}$, pudiendo ser de presentación aguda en muchos de ellos, motivo por el cual el pediatra debe conocerlos para poder sospecharlos, proveer los cuidados iniciales y coordinar el envío de las primeras muestras para estudios diagnósticos. Lo anterior resulta crucial para evitar secuelas e incluso la muerte. A pesar de esta presentación y considerando el reconocimiento de un espectro cada vez más amplio, hoy se sabe que los ECM pueden presentarse antes del parto o producir los primeros síntomas en la edad adulta.

La Pesquisa Neonatal ha cambiado el enfrentamiento de estas entidades, enfrentando a los clínicos de forma creciente con pacientes asintomáticos con un diagnóstico establecido en el período de recién nacido. En Chile, la fenilcetonuria representa el primer ECM que se diagnostica como parte del Programa de Pesquisa Neonatal junto al hipotiroidismo congénito desde el año $1992^{2}$, sin embargo, la Pesquisa Neonatal Ampliada únicamente se ofrece de forma privada, y no aún como un programa nacional.

El objetivo del presente artículo es actualizar los conceptos para lograr una adecuada aproximación al manejo inicial en pacientes con sospecha de ECM.

\section{Fisiopatología general}

Los ECM se producen por un defecto genético que afecta la función de una enzima en una vía metabólica. Consecuentemente se producirán tres efectos fisiopatológicos:

a) Acúmulo del sustrato.

b) Un déficit del producto.

c) Una activación de rutas alternativas con producción de metabolitos tóxicos.

\section{Clasificación}

Desde el punto de vista clínico es útil dividirlos en 4 grandes grupos ${ }^{3,4}$ :

\section{Síntomas agudos en el período neonatal}

Se manifiestan con síntomas inespecíficos iniciales como rechazo alimentario, vómitos explosivos, apneas centrales o episodios agudos que aparentemente ponen en peligro la vida (ALTE), progresivo compromiso de conciencia, desde el letargo y somnolencia hasta el coma profundo, convulsiones, compromiso hemodinámico y muerte. Se puede presentar también edema cerebral e incluso hemorragia intracraneana. Debe tenerse en consideración que una sepsis se puede presentar de manera similar, y que los pacientes con ECM tienen riesgo aumentado de infectarse, por lo que la presencia de infección no descarta ECM.

\section{Síntomas agudos y recurrentes de inicio más tardío}

Pueden presentarse alrededor del sexto mes de vida en relación a cambios nutricionales o la presencia de infecciones, o bien, aparecer en adolescentes o adultos jóvenes en relación a gatillantes como ingesta excesiva de proteínas, menarquia, cirugías o cualquier otro evento que produzca un stress metabólico importante. Cada episodio puede derivar en mejoría espontánea o en muerte inexplicada. Durante el período intercrisis, el paciente parece normal clínica y bioquímicamente. Frente al niño que presenta compromiso de conciencia se debe descartar un ECM, así como ante los diagnósticos de encefalitis, ataxia, jaqueca o intoxicación, especialmente cuando presenta cetoacidosis, hiperamonemia o acidosis láctica.

\section{Síntomas crónicos y progresivos}

Su presentación más habitual es a través de síntomas gastrointestinales (anorexia, vómitos), neurológicos (retardo del desarrollo psicomotor, convulsiones, trastornos motores y cognitivos) y retardo del crecimiento.

\section{Síntomas específicos y permanentes, característicos del ECM}

Hallazgos tales como miocardiopatía dilatada o hipertrófica, hepatoesplenomegalia, alteraciones del cristalino o dismorfias pueden orientar a ECM específicos. 


\section{Clínica}

La mayoría de los pediatras suelen plantearse un ECM cuando patologías más frecuentes (sepsis, inmadurez, trauma, etc.) han sido ya excluidas o cuando el diagnóstico después de un tiempo razonable de investigación aún no ha sido encontrado, lo que retrasa el diagnóstico muchas veces hasta después que el paciente ha fallecido. Debido a lo anterior, es importante recalcar que para hacer el diagnóstico de ECM no es necesario tener un extenso conocimiento de las vías metabólicas, sino más bien es importante tener un entendimiento de las diferentes manifestaciones clínicas y saber ubicarlas en las grandes agrupaciones fisiopatológicas, a través de las cuales se orientará la forma de hacer el manejo inicial.

\section{Sospecha}

Un ECM debiera ser planteado en el paciente pediátrico ante la presencia de ${ }^{5}$ :

- Un brusco deterioro del estado general y/o compromiso de conciencia, en especial si es precedida de vómitos, fiebre, ayuno, constipación o algún otro estado hipercatabólico.

- Encefalopatía rápidamente progresiva sin etiología determinada.

- Historia familiar de muerte neonatal ("muerte súbita") o consanguinidad parental.

- Apneas o distrés respiratorio.

- Características dismórficas específicas.

- Visceromegalia.

- Acidosis metabólica, hiperamonemia.

- Olor peculiar [a humedad (PKU), caramelo (Enfermedad por orina olor a jarabe de arce), a pies sudados (aciduria isovalérica)].

La clásica historia en el recién nacido es un súbito compromiso general después de una ventana de días de normalidad. Sin embargo, hay algunas excepciones que se pueden manifestar desde el primer día de vida (ej. piruvato carboxilasa y aciduria glutárica tipo II). Lo más importante será entonces el alto nivel de sospecha ante síntomas comunes para el pediatra, así como el reconocimiento de patrones de síntomas más que el hallazgo de síntomas aislados (ej: la tríada de hipotonía, apneas y espasmos en Encefalopatía Hiperglicinémica)

Después del período neonatal, habrá que sospechar un ECM cuando se observa compromiso de más de un órgano o sistema. También puede haber un curso episódico asociado a estados hipercatabólicos menores como infecciones banales, cambios nutricionales (ej: incorporación de carne a la dieta) o falla del crecimiento y/o retraso del desarrollo.

\section{Laboratorio inicial}

Es habitual contar inicialmente con exámenes generales, que pueden ser obtenidos en la mayoría de los laboratorios de hospitales en nuestro país. En la tabla 1 se pueden ver las alteraciones de laboratorio más comunmente orientadoras $^{3,6}$.

Los exámenes confirmatorios deben ser referidos rápidamente al laboratorio especializado, para lo cual la Espectrometría de Masas en Tándem o Determinación de Acilcarnitinas es muy útil. Esta requiere una simple muestra de sangre en papel filtro, a partir de la cual esposible detectar más de 30 ECM y sus formas variantes. Complementariamente, se requiere la cuantificación de aminoácidos y ácidos orgánicos en orina. En el caso de alteraciones del ciclo de la urea, la determinación de ácido orótico resulta útil para diferenciar si el defecto enzimático es mitocondrial.

\section{Muestra crítica}

En casos de sospecha de un ECM es fundamental tomar una muestra crítica, la cual servirá para precisar el diagnóstico, así como también para realizar asesoría genética ${ }^{3,7}$ (tabla 2).

Es importante destacar que en la mayoría de los ECM el diagnóstico se puede sospechar con la clínica y un laboratorio básico, permitiendo iniciar tratamientos que pueden salvar la vida del paciente. Los estudios que deben ser derivados a centros especializados nos permiten confirmación de nuestra sospecha diagnóstica, pero con una buena orientación inicial sólo deben ser solicitados en casos seleccionados. 
Tabla 1. Estudios de laboratorio sugeridos en niños con sospecha de un error congénito del metabolismo

\begin{tabular}{|c|c|c|}
\hline Examen de laboratorio & Hallazgo & Patológia posible \\
\hline Hemograma con recuento de plaquetas & Neutropenia y/o trombocitopenia & Acidurias orgánicas \\
\hline Orina completa & $\begin{array}{l}\text { Cuerpos cetónicos }(+) \text { en recién } \\
\text { nacido }\end{array}$ & Acidurias orgánicas \\
\hline Gases venosos & $\begin{array}{l}\text { Acidosis metabólica con aumento } \\
\text { del anión gap }(\geq 16)\end{array}$ & $\begin{array}{l}\text { Acidurias orgánicas (asociado a hiperamonemia } \\
\text { importante, lactacidemia, neutropenia y trombo- } \\
\text { citopenia) } \\
\text { Defectos del metabolismo de piruvato y de la cade- } \\
\text { na respiratoria (asociado a aumento lactato) }\end{array}$ \\
\hline Ácido Láctico & Elevado & $\begin{array}{l}\text { Acidurias orgánicas, enfermedades mitocondriales } \\
\text { o glucogenosis tipo I. }\end{array}$ \\
\hline Glicemia & Hipoglicemia & $\begin{array}{l}\text { Glucogenosis, galactosemias, trastorno de la beta- } \\
\text { oxidación de los ácidos grasos }\end{array}$ \\
\hline Nitrógeno ureico & Disminuido & Defectos del ciclo de la urea \\
\hline \multirow[t]{2}{*}{ Amonio* } & Aumento en período neonatal & $\begin{array}{l}\text { Déficit de piruvato carboxilasa o aciduria glutárica } \\
\text { tipo II, hiperamonemia transitoria del RN }\end{array}$ \\
\hline & $\begin{array}{l}\text { Aumento después de } 24 \text { h de } \\
\text { vida }\end{array}$ & $\begin{array}{l}\text { Defectos del ciclo de la urea, acidurias orgánicas } \\
\text { (asociado a acidosis metabólica) }\end{array}$ \\
\hline
\end{tabular}

*El rango de elevación de acidurias orgánicas y defectos del ciclo del urea suele ser de 10 a 39 veces el valor normal (VN), mientras que en las enfermedades mitocondriales suele ser de 2 a 3 veces el VN y en la hiperamonemia transitoria así como en el recién nacido prematuro el valor de amonio es de máximo 2 veces el VN.

Tabla 2. Muestra crítica en niños con sospecha de un error congénito del metabolismo

\begin{tabular}{|lll|}
\hline Muestra & Cantidad & Conservación \\
\hline Sangre para obtener suero & $2-5 \mathrm{ml}$ & Congelar a $-20^{\circ} \mathrm{C}$ \\
Gotas de sangre en papel filtro & 1 tarjeta & Secar a $\mathrm{T}^{\circ}$ ambiente, guardar en bolsa plástica y congelar a $-20^{\circ} \mathrm{C}$ \\
\hline Orina & $5-15 \mathrm{ml}$ & Congelar a $-20^{\circ} \mathrm{C}$ \\
Líquido cefalorraquídeo & $1 \mathrm{ml}$ & Congelar a $-20^{\circ} \mathrm{C}$ \\
\hline Post mortem: biopsia de hígado y músculo & 1 muestra & Congelar a $-70^{\circ} \mathrm{C}$ (hielo seco) \\
\hline
\end{tabular}

\section{Protocolo de manejo agudo general ante sospecha de $\mathrm{ECM}^{8}$}

\section{Inmediato}

- Practicar exámenes generales y tomar "muestra crítica".

- Soporte vital y estabilización clínica.

- Mantención hidroelectrolítica y del equilibrio ácido-base: corregir la acidosis metabólica con bicarbonato si el $\mathrm{pH}$ es $<7,10$ o bicarbonato $<10 \mathrm{mEq} / 1$.

\section{Evitar producción endógena de metabolitos tóxicos y favorecer anabolismo}

Régimen cero en las primeras $24 \mathrm{~h}$, suero glucosado al 10\% (según hipoglicemia o por ingesta calórica aportando $6-8 \mathrm{mg} / \mathrm{kg} / \mathrm{min}$ de carga de glucosa inicialmente). Considerar que el ayuno prolongado es perjudicial y debe ser mantenido la menor cantidad de tiempo posible.

Al segundo día aportar triglicéridos de cadena mediana para prevenir catabolismo proteico $(0,5-2 \mathrm{~g} / \mathrm{kg} /$ día $)$, siempre que se haya descartado un defecto de beta oxidación de ácidos grasos.

Cuando exista compromiso de conciencia debe instalarse un catéter central y aportar por vía parenteral calorías no proteicas a partir de glucosa $(10-15 \%)$ y lípidos $(2-4 \mathrm{~g} / \mathrm{kg} /$ día $)$ indicando insulina para favorecer anabolismo y mantener glicemia en rangos adecuados $(0,2-$ $0,3 \mathrm{U} / \mathrm{kg} / \mathrm{h})$. 
Se debe proceder así en caso de una acidosis metabólica con grave cetoacidosis o bien si el amonio sobrepasa los $300 \mathrm{ug} / \mathrm{dl}$. En este último caso, se recomienda usar benzoato de sodio o fenilbutirato como detoxificadores. Tanto los carbohidratos como los lípidos se deben dar inicialmente por vía endovenosa, pero se debe usar la vía enteral lo más pronto posible (mezclas de polímeros de glucosa y triglicéridos de cadena mediana utilizando una bomba de infusión continua). Iniciar fórmulas metabólicas específicas sólo cuando el diagnóstico haya sido confirmado. Estas deben ser iniciadas en forma paulatina por su alta osmolaridad, no sobrepasar los $450 \mathrm{mOsm} / 1$.

\section{Suplemento de sustratos}

- Administrar L-Carnitina a todos los pacientes, en dosis de $150-300 \mathrm{mg} / \mathrm{kg} /$ día por vía endovenosa u oral, ya sea en infusión continua o fraccionada en 3 dosis diarias.

- Clorhidrato de arginina al 10\% también se prescribe en todos los casos hasta no aclarar etiología (dosis: 0,6 g/ $/ \mathrm{kg}$ o $600 \mathrm{mg} / \mathrm{kg}$ / día a pasar en 90 min endovenoso).

- Las vitaminas que se suplementan son Biotina $(10 \mathrm{mg} /$ día oral o por $\mathrm{SNG})$, Tiamina (50 mg/día), Riboflavina (100 mg/día) y B12 (10 mg/día)

- Como un ejemplo, en la acidemia metilmalónica se indica vitamina B12, 1-2 mg IM. Terapias más específicas de la enfermedad se inician cuando se tenga diagnóstico definitivo y consisten básicamente en restringir los compuestos específicos involucrados en el defecto enzimático. Si los exámenes fueran negativos, a las $48 \mathrm{~h}$ aportar leche materna o fórmula de bajo contenido proteico.

\section{Remoción de sustancias tóxicas}

El tratamiento nutricional intensivo y la suplementación de sustancias descritas debieran en muchos casos mejorar el cuadro. En caso de que esto no ocurra en 24-48 h, se debe considerar la remoción de sustancias tóxicas con diálisis.

\section{Indicaciones de diálisis}

- Amonemia > a $500 \mathrm{ug} / \mathrm{dl}$ o $300 \mathrm{uM} / 1$ (hiperamonemia severa).
- Acidosis metabólica extrema.

- Compromiso de conciencia progresivo.

- Convulsiones.

- Coma.

La mejoría clínica con diálisis aparece en forma variable, a las 24-48 h en las acidurias orgánicas y, entre 3 a 7 días, en las alteraciones del ciclo de la urea.

Frente a hipoglicemia de probable origen metabólico se sugiere: a) Intentar normalizar los valores para evitar daño cerebral, recogiendo muestra basal de sangre y orina; b) Efectuar diagnóstico diferencial según presencia o ausencia de acidosis metabólica, cetosis, alcalosis, hiperamonemia; c) Comprobar tolerancia al ayuno; d) Aumentar frecuencia y volumen de hidratos de carbono.

\section{Manejo específico ECM}

A continuación se mencionan las estratégias para tratar los diferentes ECM:

\section{Evitar acumulación del sustrato}

En algunas ECM esta estrategia resulta $100 \%$ exitosa, como ocurre con la eliminación de la fenilalanina dietaria en la fenilcetonuria o de la leucina en la enfermedad de orina con olor a jarabe de arce, evitándose el retardo mental o la muerte con su inicio precoz. En otras patologías la eliminación del tóxico no es efectiva por sí sola como en la tirosinemia tipo I, donde la eliminación de tirosina no evita el hepatocarcinoma, siendo necesario el uso de NTBC para esto. En la tabla 3 se prensentan algunos ECM cuyo tratamiento se basa en la restricción del sustrato.

\section{Suplementación del producto en déficit}

Es necesaria cuando no se sintetiza la sustancia posterior al bloqueo. Ejemplos de ello son los defectos enzimáticos en el ciclo de la urea como las deficiencias de carbamil fosfato sintetasa (CPS), ornitina transcarbamilasa (OTC), arginosuccínico sintetasa (ASS) y liasa (ASL). En todas ellas, la administración de arginina es fundamental ya que se trasforma en un aminoácido esencial. 
Tabla 3. Errores congénitos del metabolismo que responden a restricción del sustrato

\begin{tabular}{|c|c|c|}
\hline Patologías & Tratamiento nutricional & Suplementación \\
\hline $\begin{array}{l}\text { Hiperfenilalaninemias } \\
\text { Fenilcetonurias }\end{array}$ & $\begin{array}{l}\downarrow \text { Fenilalanina y } \\
\uparrow \text { tirosina }\end{array}$ & L-Tirosina \\
\hline Enfermedad de la orina olor a jarabe de arce & $\downarrow$ Leucina & $\begin{array}{l}\text { Tiamina: } 100-300 \mathrm{mg} / \mathrm{día} \\
\text { L-Valina, L-Isoleucina }\end{array}$ \\
\hline Tirosinemia tipo 1a & $\begin{array}{l}\downarrow \text { Fenilalanina y } \\
\downarrow \text { tirosina }\end{array}$ & NTBC \\
\hline Acidemia isovalérica & $\downarrow$ Leucina & $\begin{array}{l}\text { L- Carnitina: } 100 \text { mg/kg/día } \\
\text { Glicina 200-400 mg/kg/día }\end{array}$ \\
\hline Aciduria 3-OH- butírica & $\downarrow$ Valina & L- Carnitina: 100 mg/kg/día \\
\hline Aciduria 3- metilglutacónica & $\downarrow$ Leucina & $\begin{array}{l}\text { L- Carnitina: } 100 \text { mg/kg/día } \\
\text { Glicina 250-400 mg/kg/día } \\
\text { Ác. Pantoténico: } 15-150 \text { mg/día }\end{array}$ \\
\hline Homocistinuria & $\begin{array}{l}\downarrow \text { Metionina } \\
\uparrow \text { Cisteína }\end{array}$ & Ácido Fólico, betaína, piridoxina: $250-750$ mg/kg/día \\
\hline
\end{tabular}

Tabla 4. Errores congénitos del metabolismo que responden a megadosis de vitaminas

\begin{tabular}{|c|c|c|}
\hline Coenzima & Enfermedad & Enzima alterada \\
\hline $\begin{array}{l}\text { Cobalamina (idealmente hidroxicobalamina) } \\
\text { Vitamina B12: 5-10 mg/día }\end{array}$ & $\begin{array}{l}\text { Acidemia metilmalónica } \\
\text { (déficit } \mathrm{Cb} \mid \mathrm{A}, \mathrm{CbLB} \text { ) }\end{array}$ & Cobalamina reductasa, adenosiltransferasa \\
\hline $\begin{array}{l}\text { Piridoxina (B6) }(50-100 \mathrm{mg} / \mathrm{d}) \\
\text { Betaína }(6 \mathrm{~g} / \mathrm{d})\end{array}$ & Homocistinuria (variante) & Cistationina B-sintetasa \\
\hline Tiamina (B1) $(10-50 \mathrm{mg} / \mathrm{d})$ & MSUD (variante) & $\begin{array}{l}\text { Complejo deshidrogenasa } \\
\text { aminoácidos ramificados }\end{array}$ \\
\hline Tetrahidrobiopterina (2 mg/kg/día) & HFA por déficit de cofactor & Dihidropterina reductasa \\
\hline Biotina $(10-20 \mathrm{mg} / \mathrm{d})$ & Deficiencia múltiple de carboxilasas & Carboxilasas \\
\hline Riboflavina (B2) (100 -300 mg/d) & Aciduria Glutárica tipo ॥ & Deshidrogensa múltiple de acil Co A \\
\hline
\end{tabular}

\section{Estimulación de vías alternativas}

La actividad de una enzima se puede afectar en el caso de que esta enzima:

a) No se sintetiza porque el gen para su transcripción está defectuoso o el transporte de la proteína no existe, o la enzima es inestable.

b) Está presente pero no se une al sustrato o lo hace defectuosamente.

c) No se une a la coenzima o hay menor cantidad de coenzima.

Las últimas alteraciones (unión a coenzima o disminución de ésta) se solucionan con la suplementación de la coenzima o de su precursor. La gran mayoría de éstas son vitaminas. Un ejemplo es la acidemia metilmalónica ocasionada por una deficiencia de la enzima meti- lamalonil CoA mutasa o del sistema cofactor. Cerca de un $50 \%$ de los casos se deben a un defecto de cofactor que es dependiente de vitamina B12. Se conoce una forma (CblA) que responde en un $100 \%$ a megadosis de Vitamina B12 no requiriendo dieta especial. En la tabla 4 se presentan otros cofactores y sus dosis como tratamiento de $\mathrm{ECM}^{3}$.

\section{Uso de drogas que eliminen el metabolito tóxico}

Consiste en unir el metabolito tóxico a otra sustancia para generar complejos no tóxicos.

El clásico ejemplo es la administración de L-carnitina en el caso de acidurias orgánicas (AO). La carnitina es un aminoácido no esencial que sintetizamos a partir de la lisina y la metionina y cuya función primaria es la in- 
Tabla 5. Errores congénitos del metabolismo y detoxificadores

\begin{tabular}{|lll|}
\hline Enfermedad & Metabolito tóxico & Tratamiento \\
Hiperglicinemia no cetócica & Glicina & Benzoato de sodio \\
Acidemia Isovalérica & Ác. Isovalérico & Glicina + Carnitina \\
Acidemia propiónica, Acidemia metilmalónica & Ácido propiónico, Ácido metilmalónico & Carnitina \\
Acidemia Glutárica tipo I & Ácido glutárico, Ácido 3-hidroxiglutárico, & Carnitina \\
Alteración ciclo urea & Lisina, Hidroxilisina y Triptófano & Benzoato Sodio + Fenilbutirato \\
\hline
\end{tabular}

corporación de ácidos grasos de cadena larga en la mitocondria para su beta oxidación. Sin embargo, en el caso de las AO, la carnitina se esterifica con los ácidos orgánicos producidos como resultado del bloqueo metabólico, convirtiéndolos en compuestos no tóxicos que son eliminados a través de la orina. En la tabla 5 se enumeran los ECM en que se usan detoxificadores ${ }^{3}$.

\section{Conclusiones}

Los ECM son enfermedades heterogéneas en cuanto a su presentación clínica y manejo, por lo que para su correcto diagnóstico se requiere en primer lugar una alta sospecha de parte del médico general y del pediatra, quien realizará la primera aproximación a este paciente. Una actitud alerta por parte del pediatra que trabaja con pacientes críticos, orientada a una pronta sospecha sindromática y al correcto y precoz inicio de un tratamiento adecuado, marcará el pronóstico de estos pacientes, evitando secuelas neurológicas y manteniéndolos con vida hasta que el manejo especializado se instaure.

\section{Referencias}

1.- Saudubray JM, Charpentier C: Clinical phenotypes: diagnosis/algorithms. En: Scriver CR, Beaudet AL, Sly WS, Valle D. The Metabolic and Molecular Bases of Inherited Disease, 8th ed. NewYork: Editorial McGrawHill 2001; 1327-406.

2.- Guthrie R, Cornejo V: Programa de detección neonatal de errores innatos del metabolismo. En: Colombo M, Cornejo V, Raimann E. Errores innatos del metabolismo del niño. Santiago: Editorial Universitaria 1999; 307-22.

3.- Raimann E, Cornejo V: Aproximación al diagnóstico y tratamiento de los errores innatos del metabolismo. En: Colombo M, Cornejo V, Raimann E. Errores innatos del metabolismo del niño. Santiago: Editorial Universitaria, 1999; 45-64.

4.- Raimann E: Diagnóstico de errores innatos del metabolismo. Rev Chil Pediatr 2008; 79: 92-5.

5.- Gupta N, Kabra M: Acute Management of Sick Infants with Suspected Inborn Errors of Metabolism. Indian J Pediatr 2011; 78: 854-9.

6.- Hudak ML, Jones MD, Brusilow SW: Differentiation of transient hyperamonemia and urea cycle defects by clinical presentation. J Pediatr 1985; 107: 712-9.

7.- Fernandes J, Saudubray JM, Huber J: Diagnostic Procedures Function Tests and Postmorten Protocol. En: Fernandes J, Saudubray JM, Van de Berghe G. Inborn Metabolic Disease Diagnosis and Treatment. New York: Editorial Springer-Verlag, 2000: 43-51.

8.- Chapman KA, Gropman A, MacLeod E, et al: Acute management of propionic acidemia. Mol Genet Metab 2012; 105: 16-25. 